\title{
Assessment of Preemptive Analgesia Efficacy in Surgical Extraction of Third Molars
}

\author{
Jorge Luiz Jacob Liporaci Junior ${ }^{1}$
}

\begin{abstract}
Summary: Liporaci Jr JLJ - Assessment of Preemptive Analgesia Efficacy in Surgical Extraction of Third Molars.
Background and objectives: Literature on preemptive analgesia is controversial. Reliability of results and difficult reproducibility of research contribute for non-elucidation of the subject. The aim of this study is to test the efficacy of oral ketoprofen (150 mg) preemptively administrated two days before third molar surgery, compared with postoperative administration in the same patient.

Methods: Thirteen patients underwent surgical removal of bilateral third molar in two separate procedures. In a random and double blind procedure, oral ketoprofen $150 \mathrm{mg}$ was administered every 12 hours two days before surgery and, after the procedure, the same drug was administered for three days. On the other side, a control (placebo) was used orally every 12 hours two days before surgery and, after the procedure, ketoprofen $150 \mathrm{mg}$ was administered every 12 hours for three days. Postoperative pain was assessed by visual analogue scale, nominal scale, and amount of rescue analgesics consumed.
\end{abstract}

Results: There was no statistically significant difference in postoperative pain between the preemptive treatment and control.

Conclusion: In this experimental model, preemptive analgesia was not effective in reducing postoperative pain in surgical extraction of third molar compared with the postoperative administration of the same drug.

Keywords: Analgesia; Molar, Third/surgery; Pain, Post-operative/prevention and control; Ketoprofen.

@2012 Elsevier Editora Ltda. All rights reserved.

\section{INTRODUCTION}

Effective treatment of postoperative pain is a major priority for clinical research in surgical practice ${ }^{1}$. Analgesia improves the quality of life postoperatively, reducing morbidity and providing greater comfort, allowing for rapid recovery and early return of patients to daily activities.

The preemptive analgesia concept was introduced by Crile at the beginning of the last century. He noted that blocking the transmission of pain before surgical incision reduced postoperative mortality. This technique was first proposed in order to prevent postoperative shock. However, later on, its proponents termed it "preemptive analgesia", as they noticed a marked decrease in the intensity and duration of postoperative pain ${ }^{2,3}$.

After major advances in the knowledge of pain physiology, preemptive analgesia has been reborn with a series of studies started by Woolf in the 80s. However, the success in experi-

Received from Faculdade de Medicina de Ribeirão Preto, Universidade de São Paulo (FMRP-USP), Brasil.

1. Maxillofacial Oral Surgeon; PhD, Faculdade de Medicina de Ribeirão Preto, Universidade de São Paulo (FMRP-USP).

Submitted on July 17, 2011.

Approved on September 12, 2011.

Correspondence to:

Flávio Teles, MD, PhD

Universidade Estadual de Ciências da Saúde de Alagoas

Jorge de Lima 113,

Trapiche da Barra

57010382 - Maceió, AL, Brazil

E-mail: flavioteles@hotmail.com mental animal models has not been repeated in human clinical models.

Several acute pain studies show a large variation in pain intensity among patients. In a review of preemptive analgesia performed by Katz et al. ${ }^{4}$, the authors report that inclusion of appropriate control groups for assessing the time of analgesic administration in relation to the surgical incision is fundamental for a better analysis of the method.

The aim of this study is to evaluate the efficacy of preemptive analgesia in surgical extraction of mandibular third molar, comparing if a non-steroidal oral anti-inflammatory (Ketoprofen $150 \mathrm{mg}$ ) administered preoperatively for two days every 12 hours is more effective in reducing or eliminating pain than the conventional protocol of postoperative administration of the same medicine every 12 hours for three days.

\section{METHOD}

After review and approval by the Ethics Committee of Hospital das Clínicas da Faculdade de Medicina de Ribeirão Preto and signing the informed consent, 14 patients were selected for the study.

The selected patients were all young adults, aged between 18 and 30 years, of both sexes, with no medical records, not using concomitant medication (except for oral contraceptive), with bilateral mandibular third molars in similar position evaluated by panoramic radiograph (Figure 1). Patients with a history of pericoronaritis or regional infections were not included in the study. 


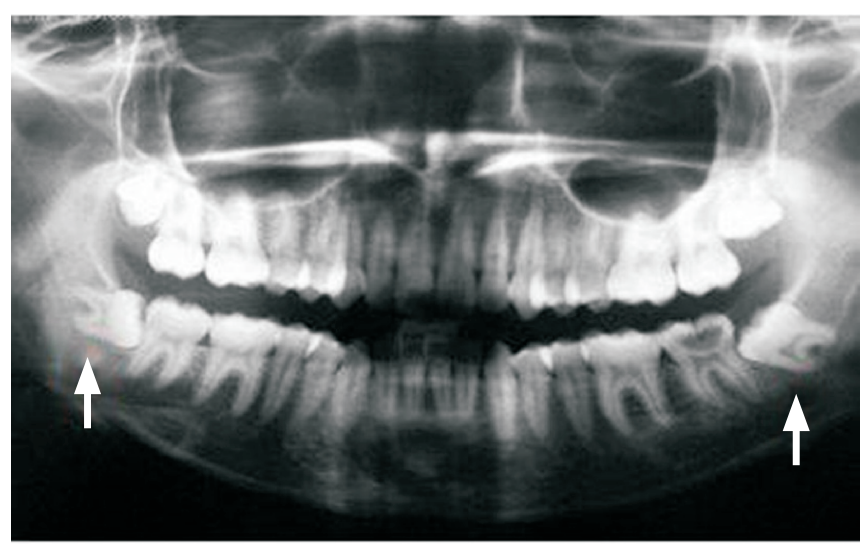

Figure 1 - Panoramic Radiograph Showing Bilateral Lower Third Molars (white arrows).

\section{Drug treatment protocol}

Patients underwent surgical removal of mandibular third molars bilaterally unerupted in two sessions with an interval of one month between them. In both procedures, patients received one drug (test or placebo), which was taken daily for two days before surgery. After surgery, the test drug (ketoprofen $150 \mathrm{mg} \mathrm{2}$. day $^{-1}$ ) was prescribed for three more days, along with a rescue analgesic (paracetamol $750 \mathrm{mg}$ ) in case of pain. Patients were fully covered in postoperative period, as placebo was used only in the two days prior to surgery in one of the procedures. Neither the surgeon nor the patient knew which drug was being used during that period, as it may be the test drug (ketoprofen 150 mg 2.day ${ }^{-1}$ ) or placebo.

\section{Surgical procedure}

The same surgeon and assistant performed the surgery, and the operative procedure did not exceed 1-hour. Following the protocol of preoperative antisepsis, local anesthesia was performed by blocking the inferior alveolar, lingual, and buccal nerves with a maximum of two tubes of $2 \%$ lidocaine with 1:50,000 noradrenaline, using carpule syringe and long needle. The surgery was performed by the classic technique of full flap, odontosection, and osteotomy to standardize the surgical trauma. After extraction of third molar, the cavity was treated with curettage, bone filing, and copious irrigation with $0.9 \%$ saline and sutured. Recommendations for the postoperative period were made, as well as for medication and completion of the pain questionnaires.

\section{Questionnaires for postoperative evaluation}

The questionnaires for pain assessment were completed at pre-established moments after surgery $(3,8,12,24,48$, and 72 hours, respectively named M1, M2, M3, M4, M5, M6) and when the patient made use of rescue analgesic (if necessary, named rescue number 1 up to rescue number 5 ). The parameters for evaluation were: 1) visual analogue scale (Huskisson ${ }^{5}$ ); 2) verbal descriptive scale (Keele ${ }^{6}$ ); 3) register of rescue analgesics consumption. For statistical analysis we used analysis of variance for parametric data and Kruskal-Wallis test for nonparametric data, and the level of significance was $p<0.05$.

\section{RESULTS}

Of the 14 patients selected, only one was excluded from the study for not taken the preoperative medication properly (patient $\mathrm{N \#} \mathrm{14).} \mathrm{Of} \mathrm{the} 13$ patients evaluated, two were males and 11 females. The patients' ages ranged from 18 to 30 years (mean age 21 years).

\section{Control vs. ketoprofen}

Comparing all surgeries in which control was used and surgeries in which ketoprofen was used, the analysis of variance showed no statistically significant difference between pain intensity at different time points $(p=0.3870)$. The results are shown in Figure 2.

\section{First surgery vs. second surgery}

Comparing the first surgery with the second surgery on the same patient, the Kruskal-Wallis test showed no statistically significant difference between pain intensity at different time points $(p=0.2897)$. The results are shown in Figure 3 .

\section{First surgery using control vs. second surgery using ketoprofen}

Comparing the first surgery using preemptive control (placebo) with the second surgery using preemptive ketoprofen in the same patient (his own control), the analysis of variance for re-

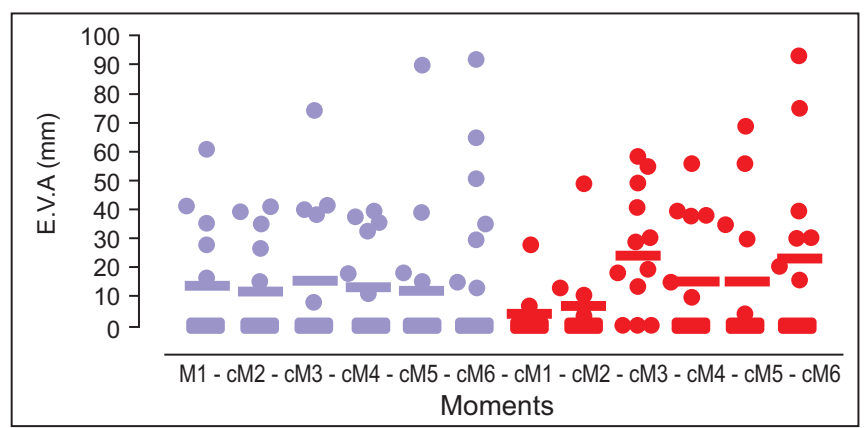

Figure 2 - Individual Values and Respective Means (horizontal line) between Control Group (left) and Ketoprofen Group Surgeries (right). 


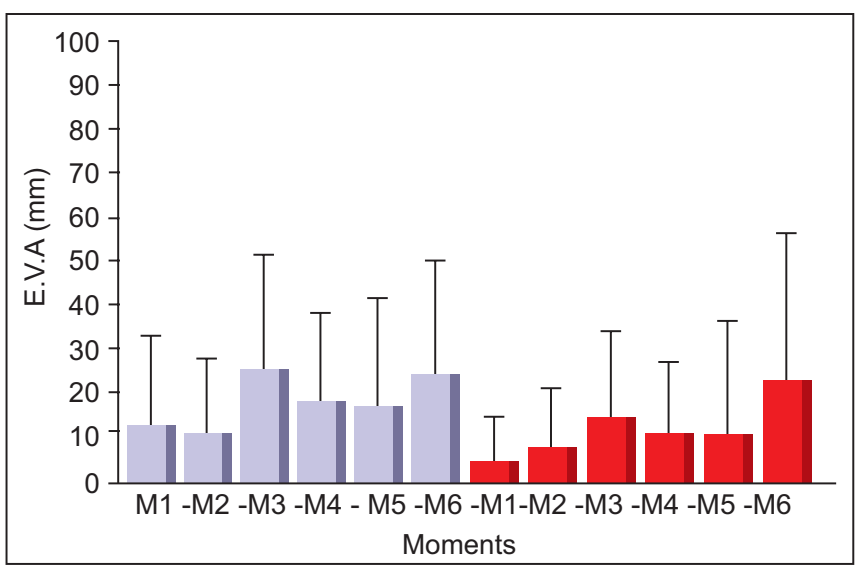

Figure 3 - Mean and Respective Standard Deviation between the First Surgery (left) and Second Surgery (right).

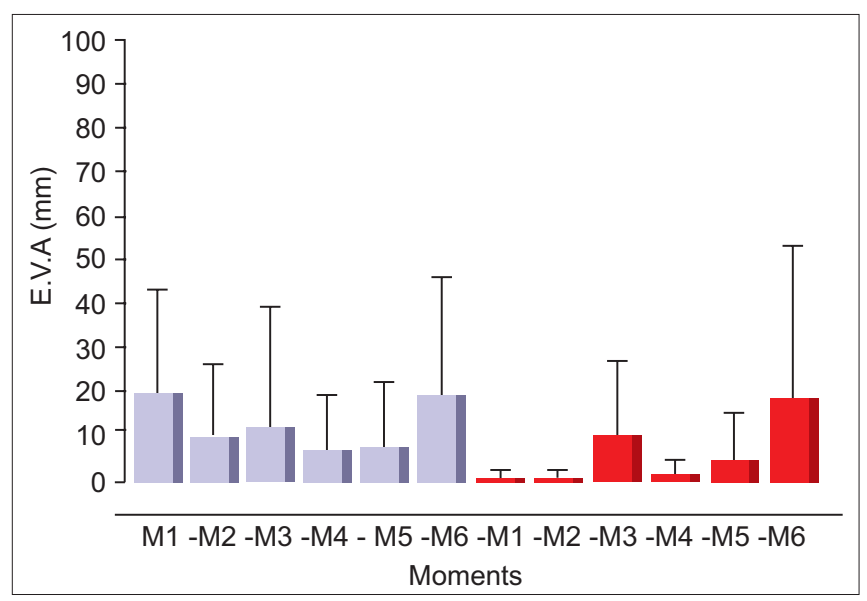

Figure 4 - Mean and Respective Standard Deviation between the First Surgery under Preemptive Control (left) and Second Surgery under Preemptive Ketoprofen (right).

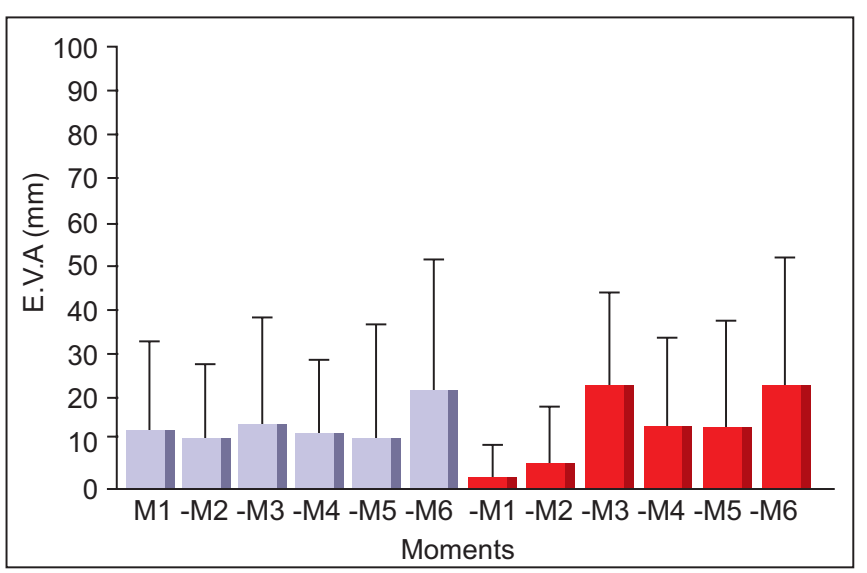

Figure 5 - Mean and Respective Standard Deviation between the First Surgery under Preemptive Ketoprofen (left) and Second Surgery under Preemptive Control (right). peated measures showed no statistically significant difference between pain intensity at different time points $(p=0.2474)$. The results are shown in Figure 4.

\section{First surgery using ketoprofen vs. second surgery using control}

Comparing the first surgery using preemptive ketoprofen with the second surgery using preemptive control on the same patient (his own control), the analysis of variance for repeated measures showed no statistically significant difference between pain intensity at different time points $(p=0.9067)$. The results are shown in Figure 5.

\section{DISCUSSION}

The results observed in this study showed no significant difference in ketoprofen used preemptively or just after surgery.

Preemptive analgesia is a controversial topic. Differences between experimental models, including different routes of administration, drug combinations, time of postoperative pain evaluation, types of surgery, concomitant use of sedation and local anesthetics that are more potent hinder the comparison between procedures.

From a conceptual standpoint, local anesthesia is in itself a preemptive analgesia, as we are blocking the nerve impulse before starting the surgical incision. Thus, we must consider that local anesthetic inhibits pain sensation both during and at the first hours after surgery. Most literature studies associate local anesthesia with the oral or parenteral drug administration regime of anti-inflammatory to evaluate analgesia, as anesthesia is necessary to perform the procedure.

In our study, we chose to use only ketoprofen 150 mg associated with local anesthesia to block the inferior alveolar, lingual and buccal nerves, using a maximum of two tubes of lidocaine $2 \%$ with epinephrine 1:100,000. Lidocaine was chosen, among other local anesthetics, because of its intermediate duration of action, as a long-lasting local anesthetic could interfere with the assessment of pain in the first 12 hours after surgery. The number of tubes was limited to avoid masking the analgesia of ketoprofen at the same initial period.

Regarding the choice of non-steroidal anti-inflammatory, we chose ketoprofen for its frequent use in several types of procedure, various routes of administration, high analgesic power and low adverse effects. This clinical efficacy is similar in intravenous, intramuscular, and rectal routes, but oral administration immediately before surgery is less effective than the intravenous (Kokki ${ }^{7}$ ). The fact that we administered ketoprofen $150 \mathrm{mg}$ two days before surgery was considering that most studies in literature using oral anti-inflammatory drugs do it with only one hour in advance. In these cases, there may 
be changes in the absorption and distribution of the drug before its clinical effect, so, we chose to anticipate the beginning of preemptive therapy.

Jung et al. ${ }^{8}$ reported that the oral administration of a nonsteroidal anti-inflammatory (Talniflumato $370 \mathrm{mg}$ ) one hour prior to surgical extraction is not more effective than the same drug administration one hour after surgery. The study evaluated only a single administration without follow-up postoperatively. In our study, the continuity of anti-inflammatory medication was justified by not leaving the patient uncovered under the risk of pain due to lack of analgesia, as the objective was to evaluate whether there is advantage to start early administration of ketoprofen $150 \mathrm{mg}$ in detriment of only postoperative administration.

Ong et al. ${ }^{9}$ reported that intravenous administration of ketorolac $30 \mathrm{mg}$ preoperatively has a preemptive effect of two additional hours of analgesia compared to the same medication administered at the end of surgery. Similar to the preceding paragraph, the authors evaluated only the first moment of post-operative pain, not assessing it afterwards or the amount of rescue analgesics. Obviously, the intravenous route has a $100 \%$ bioavailability of the drug in the circulation, which can be reduced in oral administration of the same drug. We chose the oral route to reproduce the routine clinical situation of dental offices, in which professionals who do this type of surgery rarely use the parenteral route.

Zacharias et al. ${ }^{10}$ evaluated preemptive analgesia by separating the patients into three groups: placebo, diclofenac $100 \mathrm{mg}$, and methadone $10 \mathrm{mg}$ administered orally 60-90 minutes before surgery, and found no significant difference between groups. Surgery was performed under general anesthesia and after anesthesia induction. They administered tenoxicam $20 \mathrm{mg}$ and dexamethasone $8 \mathrm{mg}$. We believe that these factors may have affected the outcome, as there is an association of different analgesics in different routes of administration, as well as central nervous system depression interfering with the perception of immediate postoperative pain. Furthermore, the study was not paired, making comparison in the same patient difficult.
Isiordia-Espinozaet et al. ${ }^{11}$ showed that the association of submucous tramadol (50 mg) applied at surgical site with ketorolac (10 mg) administered orally 30 minutes before surgery was more effective than the preemptive use of oral ketorolac (10 $\mathrm{mg}$ ) alone. This finding suggests that the balanced analgesia optimizes preemptive treatment. However, the fact that the authors used a preemptive analgesic opioid capable of sedation/depression of the central nervous system results in interference in nociceptive perception and interpretation. We decided to use only a non-steroidal anti-inflammatory to prevent such interference in our analysis.

Analgesia at hospital surgical clinic, usually, begins right in the induction of general anesthesia by the same anesthesiologist. In some cases, the surgeon requests a specific drug. During general anesthesia, a state in which the central nervous system depression prevents pain processing, the anesthesiologist usually administers an intravenous cocktail consisting of corticosteroids, opioids, and non-steroidal antiinflammatory. The surgeon, in turn, performs nerve block or infiltration of local anesthetics in the region to be operated, which complements the analgesia closing the possible pathways of nociceptive transmission.

This balanced analgesia is proven effective in preventing pain. The fact that it is administered before the surgical incision has as main justification the pharmacokinetic advantage, i.e., at the time of incision there is already an analgesic in that tissue ready to act. From that perspective, there are no controversies.

Preemptive analgesia has been investigated with the possibility of a pharmacodynamic advantage. With the onset of surgical trauma and inflammatory cascade activation (that happens even after the surgery), there is the release of inflammatory mediators and its corresponding nociceptors sensitization. The hypothesis of the anti-inflammatory administered before trauma reduce the amount of release of these mediators and the consequent peripheral and central sensitization is attractive. However, the results from literature are contradictory and more studies are needed on the subject. 


\section{REFERENCES}

1. Thomson PJ, Rood JP - Mental nerve dysfunction: a symptom of diverse mandibular disease. Dent Update. 1995 Sep; 22: 271-274.

2. Kelly DJ, Ahmad M, Brull RSJ - Preemptive analgesia I: physiological pathways and pharmacological modalities. Can J Anaesth, 2001;48:1000-1010.

3. Kelly Ahmad M, Brull RSJ - Preemptive analgesia II: recent advances and current trends. Can J Anaesth, 2001;48:1091-1101.

4. Katz J, McCartney CJ - Current status of preemptive analgesia. Curr Opin Anaesthesiol, 2002;15:435-441.

5. Huskisson EC - Measument of pain. Lancet, 1974;2:1127-1131.

6. Keele, KD - The pain chart. Lancet, 1948;2:6-8. 
7. Kokki $\mathrm{H}$ - Ketoprofen pharmacokinetics, efficacy, and tolerability in pediatric patients. Paediatr Drugs, 2010;12:313-329

8. Jung YS, Kim MK, Um YJ, Park HS, Lee EW, Kang JW - The effects on postoperative oral surgery pain by varying NSAID administration times: comparison on effect of preemptive analgesia. Oral Surg Oral Med Oral Pathol Oral Radiol Endod, 2005;100:559-563.

9. Ong KS, Seymour RA, Chen FG, Ho VC - Preoperative ketorolac has a preemptive effect for postoperative third molar surgical pain. Int $\mathrm{J}$ Oral Maxillofac Surg, 2004;33:771-776.

10. Zacharias $M$, Hunter $K M$, Baker $A B$ - Effectiveness of preoperative analgesics on postoperative dental pain: a study. Anesth Prog, 1996;43:92-6.

11. Isiordia-Espinoza MA, Pozos-Guillén AJ, Martínez-Rider R, HerreraAbarca JE, Pérez-Urizar J - Preemptive analgesic effectiveness of oral ketorolac plus local tramadol after impacted mandibular third molar surgery. Med Oral Patol Oral Cir Bucal, 2011;16:776-780. 